\title{
Methodik zur Bewertung von Effizienzgewinnen durch erhöhte Flexibilität selbstorganisierter Systeme in der Intralogistik
}

\author{
DIPL.-ING. JAN ROBERT NOPPER \\ FRAUNHOFER-INSTITUT FÜR MATERIALFLUSS UND LOGISTIK, \\ JOSEPH-VON-FRAUNHOFER-STR. 2-4, 44227 DORTMUND \\ JAN.NOPPER@IML.FRAUNHOFER.DE \\ Prof. DR. MiCHAEL TEN HOMPEL \\ FRAUNHOFER-INSTITUT FÜR MATERIALFLUSS UND LOGISTIK, \\ JOSEPH-VON-FRAUNHOFER-STR. 2-4, 44227 DORTMUND
}

\section{Zusammenfassung}

Der steigenden Dynamik und Komplexität von Materialflusssystemen kann durch die Einführung selbstorganisierter Systeme - dem Internet der Dinge in der Intralogistik - begegnet werden. Diese versprechen insbesondere durch eine erhöhte Flexibilität deutliche Effizienzgewinne über den Lebenszyklus. Der vorliegende Artikel schlägt eine Methodik zur Bewertung der erhöhten Flexibilität vor, illustriert diese anhand eines einfachen Beispiels und zeigt weiteren Forschungsbedarf auf. Die vorgeschlagene Methodik beruht auf einer Betrachtung der durch Flexibilität beeinflussten Auszahlungen im Lebenszyklus mit Hilfe einer dynamischen Optimierung.

\section{Abstract}

Self-organization and the Internet of Things in facility logistics provide a means to deal with dynamic and complex material handling systems. This is mainly due to an increase in flexibility, which can provide significant efficiency gains over the life-cycle of the material handling system. This article suggests a methodology to evaluate the advantages of flexibility over the life-cycle, illustrates its usage, and specifies further research needs. The proposed methodology is based on a dynamic optimization of payoffs during the life-cycle. For that, all payoffs which are influenced by the flexibility of the material-handling system are considered.

Keywords:

Internet of Things; Flexibility; Measurement of flexibility; Dynamic optimization; Life-cycle costs; Net present value.

\section{Einführung}

Die Anforderungen an intralogistische Materialflusssysteme und deren Steuerung sind in den letzten Jahren deutlich gestiegen [tenHompel08a]. Wesentliche Ursachen dafür sind dynamische Schwankungen von Nachfrage und Angebot, die räumliche Verlagerung von Produktionsstätten in Zusammenhang mit der Globalisierung und ein steigender Bedarf nach individualisierten Produkten ([tenHompel08a], [deMeyer98], [Schuh06], [Strüven04]).

Ein wesentlicher Ansatz zum Umgang mit steigender Dynamik und Komplexität ist der Einsatz dezentraler, selbstorganisierter Systeme - oft verknüpft mit dem Begriff des Internets der Dinge. Selbstorganisierte Systeme sind dabei sowohl in der Intralogistik ([Bullinger07], [tenHompel06], [Günthner08a], [Günthner08b], [Kohagen08]), als auch in übergreifenden Netzen und Supply Chains Gegenstand aktueller Forschung ([Bretzke06], [Jedermann06], [Scholz-Reiter07]). Die technische Umsetzung dezentraler selbstorganisierter Systeme in der Intralogistik ist weit fortgeschritten: [tenHompel08b] zeigen, dass sich durch einen agentenbasierten Ansatz die Komplexität der Steuerung von Gepäckförderanlagen bei gleichbleibender logistischer Leistung etwa um einen Faktor 10 verringern lässt. Das Internet der Dinge wurde bereits erfolgreich 
zur Steuerung realer Anlagen eingesetzt, unter anderem am openID-Center des Fraunhofer-Instituts für Materialfluss und Logistik in Dortmund, sowie am Lehrstuhl fml der TU München.

Für den praktischen Einsatz in der Industrie ist neben dem Nachweis der technischen Machbarkeit auch eine Quantifizierung der erreichbaren Effizienzgewinne notwendig. Diese werden insbesondere durch die gegenüber herkömmlichen Anlagen erhöhte Flexibilität selbstorganisierter Anlagen erwartet ([tenHompel06], [Günthner08b], [Kohagen08]). Die vorliegende Arbeit soll durch die Entwicklung einer stringenten Methodik zur Quantifizierung von Effizienzgewinnen durch Flexibilität in der Intralogistik einen Beitrag zur Anwendung des Internets der Dinge in der Praxis leisten.

Dafür wird in Abschnitt 2 zunächst der Stand der Wissenschaft zur Bewertung von Flexibilität und Wandelbarkeit von Materialfluss- und Fertigungssystemen zusammengefasst. Aufbauend darauf wird in Abschnitt 3 eine Methodik zur Quantifizierung der Effizienzgewinne durch Flexibilität intralogistischer System vorgeschlagen, die in Abschnitt 4 anhand eines einfachen Beispiels illustriert wird. Abschnitt 5 fasst wesentliche Ergebnisse zusammen und identifiziert weiteren Forschungsbedarf.

\section{Bewertung von Flexibilität und Wandelbarkeit intralogistischer Systeme}

Die Definition, Beschreibung und Messung von Flexibilität in Fertigungssystemen ist bereits seit langem Gegenstand wissenschaftlicher Untersuchungen. [Slack83] identifiziert drei bestimmende Faktoren im Zusammenhang mit Flexibilität:

- $\quad$ Flexibilitätsart (z.B. Produktmix, Volumen)

- $\quad$ Reichweite der Änderungen je Flexibilitätsart im Sinne möglicher Systemzustände

- $\quad$ Kosten und Zeitbedarf je Zustandswechsel

Dabei definiert er, dass ein System, bei dem reibungslose und günstige Zustandswechsel möglich sind, flexibler ist als ein System, bei dem gleiche Wechsel mit höheren Kosten verbunden sind. Weiter führt er aus, dass Kosten und Zeitbedarf in diesem Zusammenhang austauschbare Größen sind - gewissermaßen 'Reibungselemente der Zustandsänderung'. Trotz zahlreicher Versuche, darauf aufbauend einen umfassenden und allgemein anerkannten Flexibilitätsbegriff zu finden (vgl. [Sethi90], [Koste99]), konnte bisher in vielen Bereichen keine Übereinstimmung gefunden werden ([Gupta93], [Bengtsson02], [Schuh06]).

Allerdings stimmen die meisten Autoren darin überein, dass

- $\quad$ eine Unterscheidung in Flexibilitätsart, Reichweite möglicher Zustände je Flexibilitätsart und Aufwand für eine Änderung des Zustandes notwendig ist (z.B. [Slack83], [Sethi90], [Upton94], [Chryssolouris96], [Möller08]) und dass

- $\quad$ Flexibilität besser über eine Ersatzgröße (z.B. eine monetären Größe) zu messen ist als über eine direkte Größe ([Gupta93], [Schuh06], [Möller08]). Abweichende Methoden wie die Messung der 'Entropie' eines Fertigungssystems ([deToni98], [Shuiabi05], [Kumar87]) scheinen dagegen für eine a priori Bewertung flexibler Systeme ungeeignet (vgl. dazu auch [Corbey91]).

Für weitergehende Untersuchungen ist es sinnvoll, die Definition und Quantifizierung von Effizienzgewinnen durch Flexibilität an die jeweilige Situation und den Nutzer anzupassen [Gupta93]. Für Materialflusssysteme gibt es bisher noch wenige Arbeiten zur Bewertung von Effizienzgewinnen durch Flexibilität. Zu erwähnen sind jedoch diverse Arbeiten aus dem Fertigungsbereich, die die Flexibilität von Materialflusssystemen zwar ansprechen (z.B. [Chen96]), dabei aber wenig auf die spezifischen Charakteristika von Materialflusssystemen eingehen. Wesentlich weiter gehen die Arbeiten von [Günthner02], [Heinecker06] und [Wilke06]. Diese identifizieren als wesentliche Flexibilitätsarten für Materialflusssysteme in der Intralogistik Fördergut-, Layoutund Durchsatzflexibilität und schlagen hierfür jeweils qualitative Bewertungsmethoden mit Hilfe von Nutzwertanalysen vor. Grundsätzlich wird hier zwischen der Wandelbarkeit und der Flexibilität eines Materialflusssystems unterschieden. Erstere bezeichnet insbesondere die Möglichkeit struktureller Anpassungen eines Materialflusssystems, die als eine erweiterte Flexibilität verstanden wird (vgl. [Wilke06]). Andere Autoren bewerten die Flexibilität eines Materialflusssystems qualitativ als Teil des Selbststeuerungsgrads eines Systems ([Böse07] und [Windt08]). Bisher existiert jedoch noch keine stringente quantitative Bewertungsmethodik für Effizienzgewinne durch Flexibilität in der Intralogistik und insbesondere keine geeignete Methodik, die auf die Bewertung selbstorganisierter Systeme zugeschnitten ist. 


\section{Eine Methodik zur quantitativen Bewertung von Effizienzgewinnen durch Flexibilität}

Daher wurde am Fraunhofer IML eine Methodik entwickelt, die geeignet ist, Effizienzgewinne durch erhöhte Flexibilität intralogistischer Systeme zu quantifizieren (Abbildung 1).

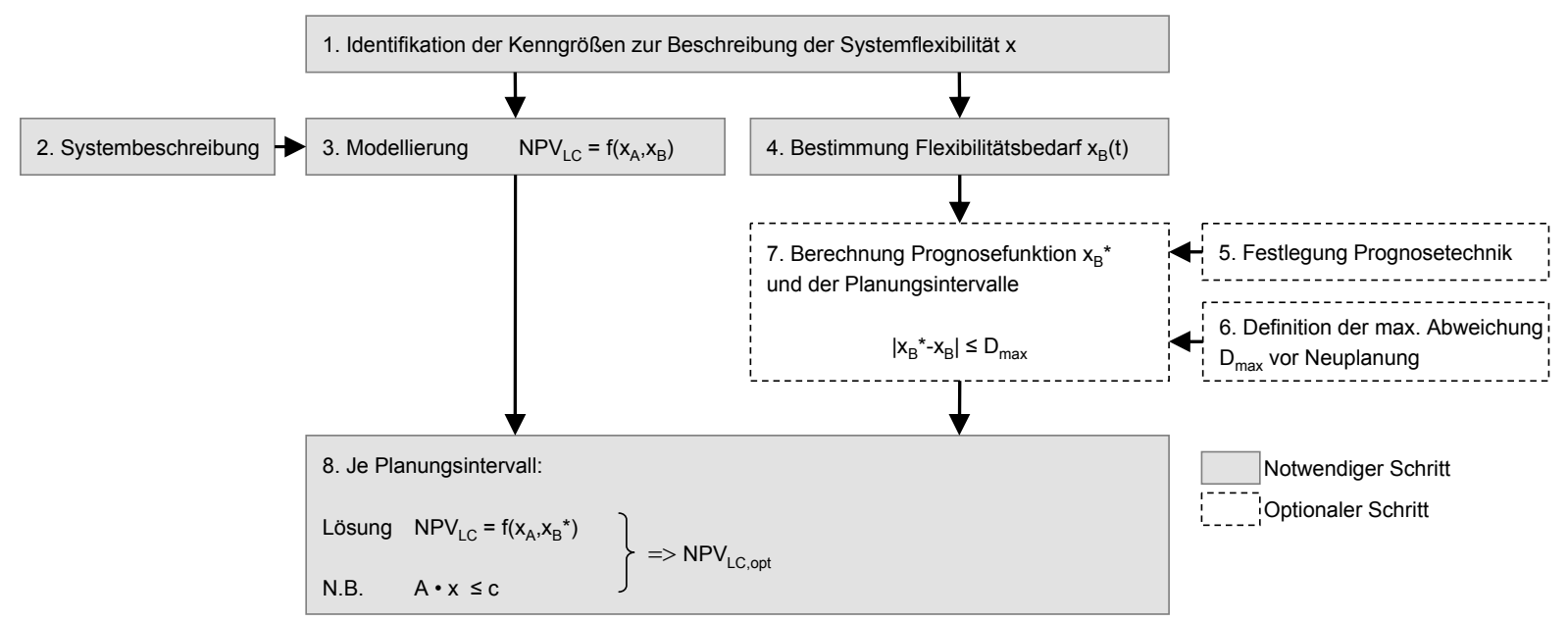

Abbildung 1: Überblick über die vorgeschlagene Methodik zur Quantifizierung von Effizienzgewinnen durch Flexibilität in der Intralogistik

Im Sinne der angestrebten Ersatzmessung werden Effizienzgewinne durch Flexibilität in Geldeinheiten ausgedrückt, wodurch Methoden der Investitionsrechnung direkt anwendbar sind. Dies hat zudem den Vorteil, dass eine Beurteilung der Ergebnisse durch Entscheidungsträger in der Praxis vereinfacht wird. Methodisch werden die durch die Flexibilität der Anlage beeinflussten Auszahlungen betrachtet. Sind diese bei gegebenen Rahmenbedingungen für ein System A geringer als für ein System B, so ist System A das flexiblere. Die Methodik besteht aus acht Teilschritten, die im Folgenden näher erläutert werden.

Schritt 1: Identifikation der Kenngrößen zur Beschreibung der Systemflexibilität

Zunächst sind die zur Beschreibung der Flexibilität des Bewertungsobjekts relevanten Kenngrößen zu bestimmen. Die Messung von Flexibilität für intralogistische Systeme erfolgt dabei in Anlehnung an [Günthner02], [Heinecker06] und [Wilke06] entlang der Dimensionen Durchsatz, Layout und Fördergut. Deren Änderung ist über geeignete messbare Größen auszudrücken, die zu einem Beschreibungsvektor $x$ zusammengefasst werden. Es ist darauf zu achten, dass die Komponenten von $x$ linear unabhängig sind: Sei beispielsweise das zu betrachtende System eine Gepäckförderanlage, bei der die Anzahl der zu transportierenden Gepäckstücke (Durchsatz) sowie die Anzahl und Lage der Check-In Schalter und Gates (Layout) jeweils abhängig ist von der Anzahl der Passagiere. Falls die Art der Gepäckstücke im Zeitverlauf gleich bleibt (Fördergut), können alle notwendigen Änderungen des Systems im Lebenszyklus alleine durch die Anzahl der Passagiere beschrieben werden, $x$ ist in diesem Fall also eindimensional zu wählen.

Schritt 2: Systembeschreibung

Anschließend sind alle zu untersuchenden Varianten des Systems technisch zu beschreiben. In der Regel ist es sinnvoll, mehr als eine Variante des Systems (beispielsweise hinsichtlich der Steuerung) zu berücksichtigen, da sich gerade aus dem Vergleich verschiedener Varianten wesentliche Erkenntnisse ergeben.

\section{Schritt 3: Modellierung der Auszahlungen im Lebenszyklus}

Für jede Variante ist nun basierend auf der technischen Beschreibung eine Bewertung der kumulierten Auszahlungen über den Lebenszyklus in Abhängigkeit von der tatsächlichen Systemgröße $x_{A}(t)$, der Änderung der Systemgröße $\mathrm{u}_{\mathrm{j}}$ und dem Bedarf an Systemgröße $x_{B}(t)$ für alle Perioden vorzunehmen.

Hierfür sind mehrere Varianten denkbar, insbesondere eine Bewertung mit Hilfe von Realoptionen (vgl. [Copeland98], [Bengtsson01], [Möller08], [Hellingrath08]) oder über die Kapitalwertmethode (vgl. [Hirschmeier05], [Okujava06]). 
Im Fall der Kapitalwertmethode lassen sich die für die Flexibilität relevanten kumulierten Auszahlungen oft in der Form

$$
N P V_{L C}=\sum_{j=1}^{N} e^{-r_{j} \cdot t_{j}} \cdot\left[I\left(u_{j}\right)+C\left(x_{A, j}, x_{B, j}\right)+O\left(x_{A, j}\right)\right]
$$

darstellen. Dabei bezeichnet $N P V_{L C}$ den Kapitalwert über den Lebenszyklus, $N$ die Anzahl der Perioden, $r_{j}$ die Kapitalkosten in Periode $j, t_{j}$ die reale Zeit in Periode $j, x_{A, j}$ und $x_{B, j}$ die tatsächliche Systemgröße und den Bedarf an Systemgröße in der Periode $j, u_{j}$ den Zuwachs von $x_{A, j}$ zu Beginn der Periode $j, I$ die Auszahlungen in Folge von Investitionen, $C$ die Auszahlungen in der Periode $j$, die durch eine im Vergleich zum Bedarf zu geringe Systemgröße verursacht werden, beispielsweise durch manuelle Ersatztransporte oder Umsatzverluste (englisch congestion costs, vgl. [Freidenfels81], S. 189 ff.) und $O$ die Auszahlungen in der Periode $j$, die zur Aufrechterhaltung des Betriebs notwendig sind.

\section{Schritt 4: Bestimmung des tatsächlichen Flexibilitätsbedarfs}

Im nächsten Schritt ist für jede Dimension von $x$ der Bedarf $x_{B}(t)$ zu bestimmen. Dieser kann beispielsweise auf historischen Werten oder Prognosewerten beruhen, wobei $x_{B}$ auch als stochastische Funktion modelliert werden kann. Mit Hilfe einer Sensitivitätsanalyse kann der Einfluss der Wachstumsdynamik auf den Wert der Flexibilität bestimmt werden.

\section{Schritte 5, 6, 7 (optional): Ableitung des prognostizierten Flexibilitätsbedarfs}

Für die nachfolgende Optimierung kann ein vom realen Bedarf $x_{B}$ abweichender prognostizierter Bedarf $x_{B}{ }^{*}$ verwendet werden. Damit ist es möglich, den zusätzlichen Wert der Flexibilität zu erfassen, der sich durch ungenaue Prognose der Zukunft ergibt. Dadurch kann beispielsweise untersucht werden, wie sich verschiedene Prognoseverfahren (vgl. [Schlittgen01]) bei gegebenem Bedarf $x_{B}$ auf den Wert der Flexibilität auswirken. Falls im Zeitverlauf die Differenz zwischen $x_{B}$ * und $x_{B}$ einen zulässigen Wert $D_{\max }$ überschreitet, wird eine Neuplanung vorgenommen. Damit lässt sich der gesamte Betrachtungszeitraum in eine bis mehrere Planungsperioden unterteilen. Im Falle von $x_{B}{ }^{*}=x_{B}$ für alle Zeiten $t_{j}$ gibt es also genau eine Planungsperiode.

\section{Schritt 8: Berechnung der kumulierten Auszahlungen über den Lebenszyklus}

Grundsätzlich wird angenommen, dass ein Entscheidungsträger im Rahmen der vorliegenden Informationen optimale Investitionszeitpunkte und -größen bezüglich der kumulierten Auszahlungen $N P V_{L C}$ wählt. Dafür muss bei gegebenem $x_{B}(t)$ (bzw. $x_{B}^{*}(t)$ ) je Variante folgendes Optimierungsproblem gelöst werden:

$$
N P V_{L C}=\sum_{j=1}^{N} g_{j}\left(x_{A, j-1}, u_{j}, t_{j}\right) \rightarrow \min
$$

mit einer abgezinsten Auszahlung je Periode (im Falle der Kapitalwertmethode)

$$
g_{j}\left(x_{A, j-1}, u_{j}, t_{j}\right)=e^{-r_{j} \cdot t_{j}} \cdot\left[I\left(u_{j}\right)+C\left(x_{A, j}, x_{B, j}\right)+O\left(x_{A, j}\right)\right]
$$

unter der dynamischen Nebenbedingung

$$
x_{A, j}=f\left(x_{A, j}, u_{j}\right)=x_{A, j-1}+u_{j}
$$

und den statischen Nebenbedingungen

$$
\begin{aligned}
& x_{A, 0}=\text { geg. } \\
& x_{A, j} \in \Xi_{j} \\
& u_{j} \in \Omega_{j}\left(x_{A, j-1}\right)
\end{aligned}
$$


Dabei macht es in der Regel Sinn, für die Definitionsbereiche die maximal erreichbare Größe zu wählen:

$$
\begin{aligned}
& \Xi_{j}=\left[0, \max _{j}\left(x_{B, j}\right)\right] \\
& \Omega_{j}\left(x_{A, j-1}\right)=\left[-x_{A, j-1}, \max _{j}\left(x_{B, j}\right)-x_{A, j-1}\right]
\end{aligned}
$$

Aufgrund der Struktur der dynamischen Nebenbedingung $f$ und dem abgeschlossenen Definitionsbereich von $x_{A, j}$ und $u_{j}$ hat das Problem eine optimale Lösung, die mit Hilfe des BELLMANschen Optimalitätsprinzips gefunden werden kann ([Neumann77], [Domschke05]). Es handelt sich um eine Aufgabe der (ggf. stochastischen) dynamischen Optimierung.

Abbildung 2 veranschaulicht die vorgeschlagene Methodik für eindimensionale $x$. Die durchgezogene Linie stellt dabei den realen Bedarf $x_{B}$ für den Betrachtungszeitraum dar. In Planungsperiode 1 wird nun für $j=[0,7]$ ein konstanter Bedarf $x_{B}{ }^{*}$ angenommen.

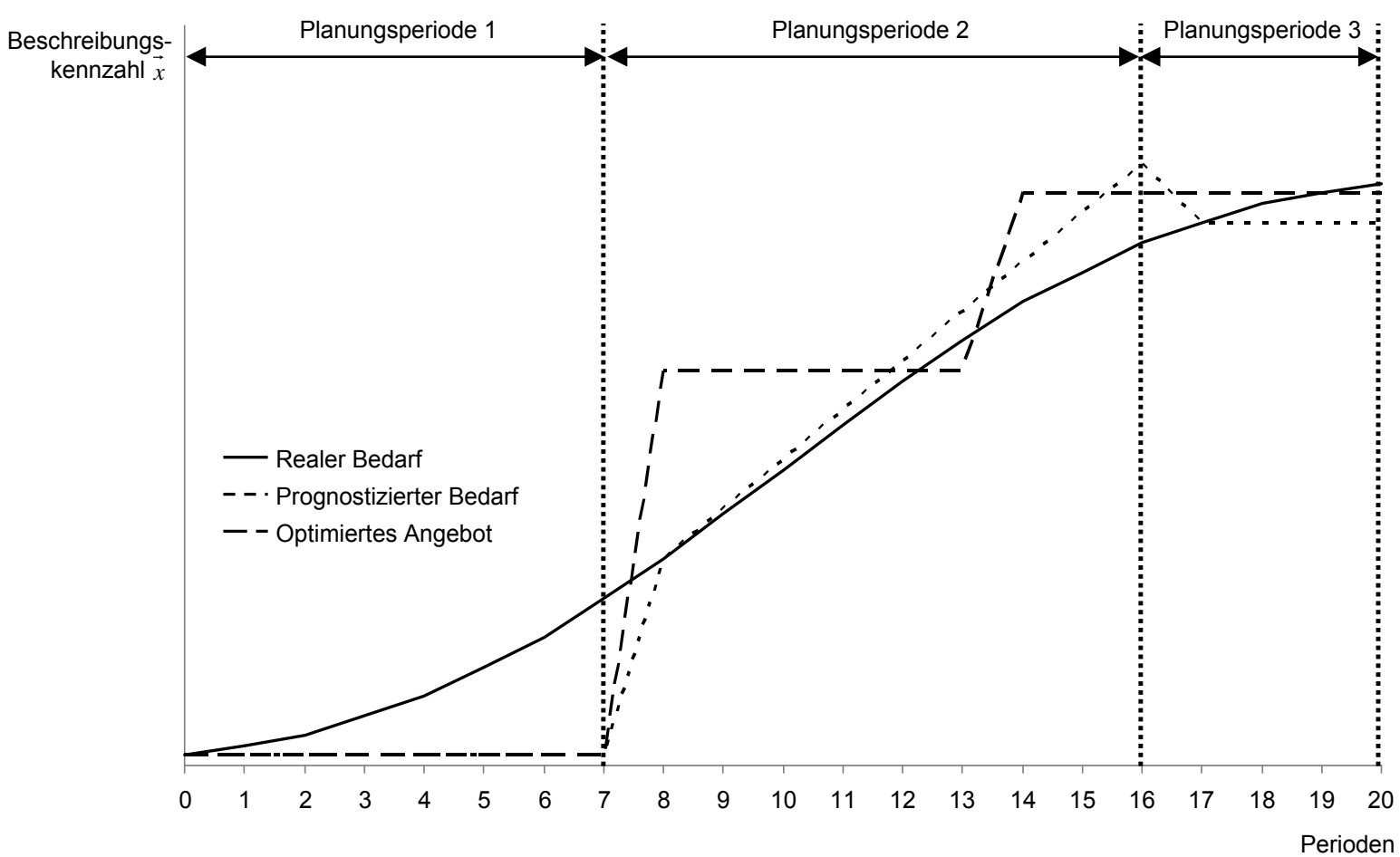

Abbildung 2: Bedarfs- und Angebotsfunktion für vorgeschlagene Methodik

Da nach Periode 7 der Unterschied zwischen $x_{B}{ }^{*}$ und $x_{B}$ größer wird als $D_{\max }$, wird neu geplant und $x_{B}{ }^{*}$ als linear steigend angenommen. Nachdem damit nach Periode $15 D_{\max }$ erneut überschritten wird, erfolgt eine weitere Neuplanung. Hierbei wird $x_{A}$ bzw. $u_{j}$ jeweils für $x_{B}{ }^{*}$ optimiert.

\section{Beispielhafte Anwendung der Methodik}

Zur Verdeutlichung der Funktionsweise der Methodik wird diese im Folgenden auf ein stark vereinfachtes Beispiel angewandt. Betrachtungsgegenstand ist ein fahrerloses Transportsystem (FTS) in einem Produktionswerk, bei dem die Produktionskapazität des Werkes den Transportbedarf bestimmen soll. Dieser kann entweder durch das FTS oder durch einen manuellen Ersatztransport geleistet werden, falls die maximale Förderleistung des FTS zu gering ist. Alle Werte seien unterjährig konstant, zu Beginn jeden Jahres kann eine Anpassung der Förderleistung des FTS durch Einbringung zusätzlicher fahrerloser Transportfahrzeuge (FTF) vorgenommen werden. Dabei soll ein konventionelles, zentral gesteuertes System mit einem selbstorganisierten System verglichen und Effizienzgewinne durch erhöhte Flexibilität quantifiziert werden. 
Schritt 1: Identifikation von Kenngrößen zur Beschreibung der Systemflexibilität

Für das gegebene Beispiel seien Layout und Durchsatz der eingesetzten Fördertechnik direkt abhängig von der Produktionskapazität $x$. Das Fördergut sei konstant für den gesamten Betrachtungszeitraum. Die einzige zur Beschreibung der notwendigen Transportleistung relevante Größe für das System ist damit $x$.

\section{Schritt 2: Systembeschreibung}

Für das System gebe es zwei mögliche Konzepte (konventionell und selbstorganisiert). Die technischen Eigenschaften beider Systeme sind in Tabelle 1 zusammengefasst.

Tabelle 1: Gewählte Parameter für illustrative Beispiel

\begin{tabular}{lrr}
\hline Parameter & Konventionell & Selbstorganisiert \\
\hline Lebensdauer der Anlage & 20 Jahre & 20 Jahre \\
Kapitalkosten & $10 \%$ & $10 \%$ \\
Produktionskapazität (Startzeitpunkt) & $0 \mathrm{St} . / \mathrm{Tag}$ & $0 \mathrm{St} . / \mathrm{Tag}$ \\
Produktionskapazität (Endzeitpunkt) & $1.000 \mathrm{St} . / \mathrm{Tag}$ & $1.000 \mathrm{St} . / \mathrm{Tag}$ \\
Wachstumsfunktion Produktionskapazität & linear & linear \\
Notwendige FTF je Produktionskapazität & $1 \mathrm{FTF} / 100 \mathrm{St}$. & $1 \mathrm{FTF} / 100 \mathrm{St}$ \\
Kosten pro FTS inkl. Steuerung & $100.000 \mathrm{GE}$ & $100.000 \mathrm{GE}$ \\
Fixkosten je Systemanpassung & $100.000 \mathrm{GE}$ & $30.000 \mathrm{GE}$ \\
Betriebskosten/Investition in FTS (ohne Fixkosten) & $15 \%$ & $15 \%$ \\
Arbeitstage pro Jahr & 200 & 200 \\
Transportmehrkosten je Stück bei manuellem Transport & $5 \mathrm{GE}$ & $5 \mathrm{GE}$ \\
\hline
\end{tabular}

Der einzige Unterschied zwischen beiden Systemen sind also die fixen Investitionskosten, die je Systemanpassung unabhängig von der Größe der Anpassung anfallen. Das selbstorganisierte System ist also in diesem Fall das flexiblere, da die von der Größe der Anpassung unabhängigen fixen Investitionskosten geringer sind.

Schritt 3: Modellierung der Auszahlungen im Lebenszyklus

Mit den Werten aus Tabelle 1 können die kumulierten Auszahlungen im Lebenszyklus mit Hilfe der Kapitalwertmethode wie folgt beschrieben werden:

$N P V_{L C}=\sum_{j=1}^{21} e^{-0,1 \cdot(j-1)} \cdot\left[I\left(u_{j}\right)+C\left(x_{A, j}, x_{B, j}\right)+O\left(x_{A, j}\right)\right]$

mit

$I_{\text {konv. }}\left(u_{j}\right)=\left\{\begin{array}{cc}1.000 \cdot u_{j}+100.000 & \forall\left(u_{j}>0\right) \\ 0 & \forall\left(u_{j}=0\right) \\ 100.000 & \forall\left(u_{j}<0\right)\end{array}\right.$

$I_{\text {selbst. }}\left(u_{j}\right)=\left\{\begin{array}{cc}1.000 \cdot u_{j}+30.0000 & \forall\left(u_{j}>0\right) \\ 0 & \forall\left(u_{j}=0\right) \\ 30.000 & \forall\left(u_{j}<0\right)\end{array}\right.$

$C\left(x_{A, j}, x_{B, j}\right)=\left\{\begin{array}{cc}1.000 \cdot\left(x_{B, j}-x_{A, j}\right) & \forall\left(x_{B, j}>x_{A, j}\right) \\ 0 & \forall\left(x_{B, j} \leq x_{A, j}\right)\end{array}\right.$

$O\left(x_{A, j}\right)=150 \cdot x_{A, j}$

Schritt 4: Bestimmung des Flexibilitätsbedarfs

In vorliegendem Beispiel handelt es sich beim Bedarf um eine deterministische Funktion. Diese kann in Abhängigkeit von Periode $j$ berechnet werden über $x_{B}(j)=50(j-1)$. 
Schritte 5, 6, 7: Ableitung des prognostizierten Flexibilitätsbedarfs

Der Unterschied zwischen $x_{B}$ und $x_{B}{ }^{*}$ wird in diesem Beispiel nicht betrachtet, d.h. $x_{B}(t)$ sei dem Entscheider für alle $t$ bekannt.

\section{Schritt 8: Berechnung der Lebenszykluskosten}

Das zu lösende Optimierungsproblem lässt sich also wie folgt darstellen:

$N P V_{L C} \rightarrow \min$

unter der dynamischen Nebenbedingung

$$
x_{A, j}=x_{A, j-1}+u_{j}
$$

und den statischen Nebenbedingungen

$$
\begin{aligned}
& x_{A, 0}=0 \\
& x_{A, j} \in[0,100,200, \ldots, 1000] \\
& u_{j} \in\left[0,1000-x_{A, j-1}\right]
\end{aligned}
$$

Dieses Problem kann durch eine leichte Abwandlung des Algorithmus aus [Neumann77] (S. 39 ff.) gelöst werden kann. Die Diskretisierung von $x_{j}$ und $u_{j}$ wurde zu 100 Einheiten Produktionskapazität gewählt, da für das zu Grunde liegende System Anpassungen nur durch Zusatz einzelner FTF oder Vielfachem davon durchgeführt werden können, die jeweils den Transportbedarf von 100 Einheiten Produktionskapazität pro Tag abdecken.

Abbildung 3 zeigt das Ergebnis der Optimierung für beide Systeme. Es ist zu erkennen, dass im Optimum des selbstorganisierten Systems mehrere kleine Anpassungen vorgenommen werden, wohingegen im Falle des konventionellen Systems weniger und dafür größere Anpassungen vorgenommen werden. Grundsätzlich entstehen im Fall $x_{B}>x_{A}$ unnötige Auszahlungen in Folge zu geringer Transportleistung des FTS und im Fall $x_{B}<x_{A}$ unnötige Auszahlungen in Folge zu hoher Transportleistung des FTS. Die entstandenen kumulierten Auszahlungen über den gesamten Lebenszyklus können nun detailliert analysiert werden.

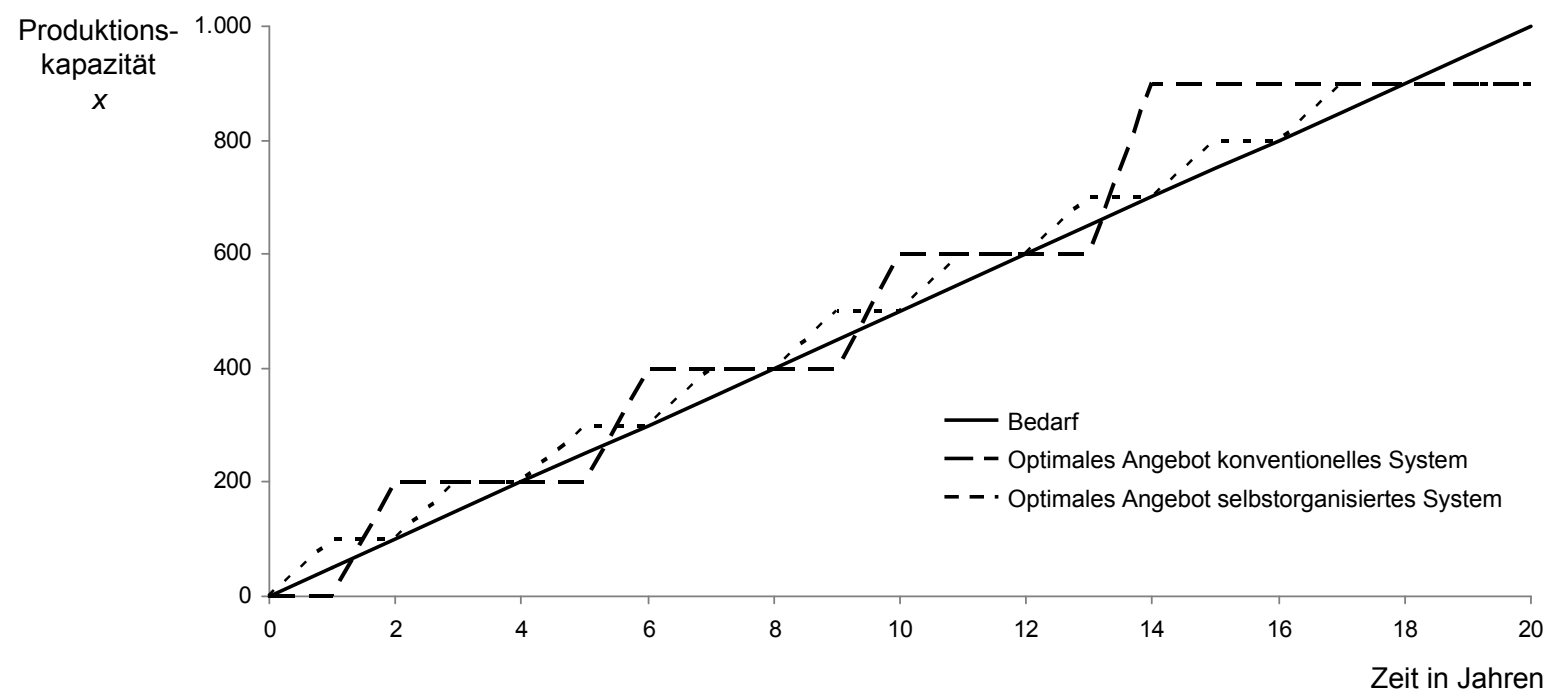

Abbildung 3: Optimale Kapazitätsanpassungen beider Varianten für das illustrative Beispiel

Abbildung 4 zeigt die Ergebnisse für das Beispielsystem. Für das betrachtete Beispiel ist das selbstorganisierte System bezüglich der kumulierten Auszahlungen etwa 16\% günstiger als das konventionelle System. Es ist beachtenswert, dass die Einsparung von fixen Investitionskosten nur knapp 40\% des Unterschieds zwischen beiden Systemen ausmacht, obwohl dies der einzige Unterschied zwischen den Systemen ist (Tabelle 1). Zusätzlich entstehen geringere Auszahlung zur Aufrechterhaltung des Betriebs und aufgrund zu geringer 
Transportleistung des FTS, da sowohl die durchschnittliche Größe des FTS als auch der Bedarf für manuelle Ersatztransporte in Folge von Unterdeckungen für das selbstorganisierte Systems geringer sind als die des konventionellen Systems. Der Unterschied der variablen Investitionskosten trotz gleicher Größe für $j>16$ ergibt sich durch die späteren Investitionszeitpunkte im Falle des selbstorganisierten Systems. Im gewählten Beispiel verursacht das selbstorganisierte System damit in jeder Komponente geringere kumulierte Auszahlungen. Das ist im Allgemeinen nicht der Fall, zeigt aber, dass ein reiner Vergleich der eigentlichen Investitionskosten zu einer deutlichen Unterschätzung der Vorteile flexibler Systeme führen kann.

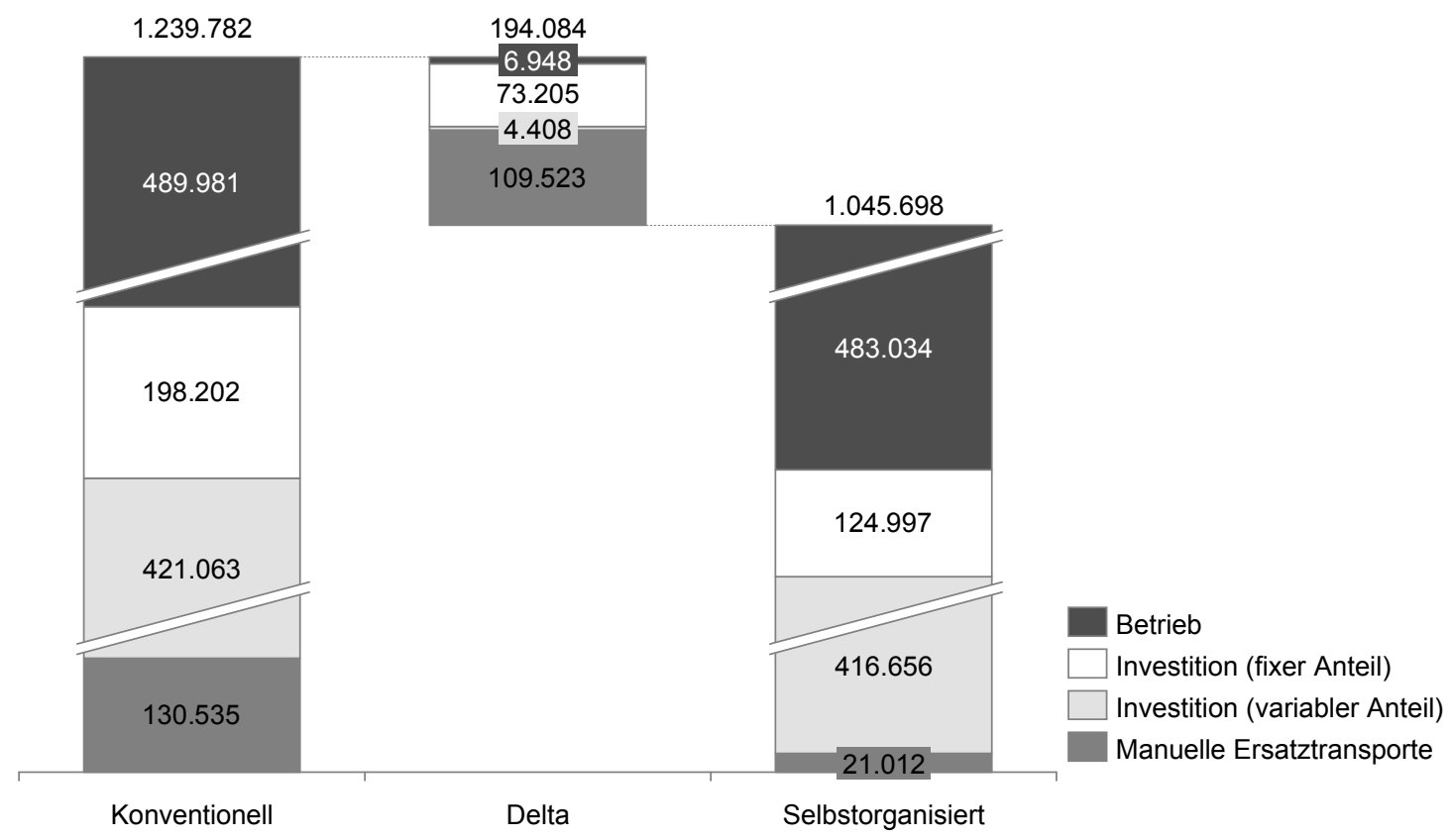

Abbildung 4: Kumulierte Auszahlungen im Lebenszyklus für das Beispielsystem

Abbildung 5 zeigt für das gegebene System die Abhängigkeit des Unterschiedes in den kumulierten Auszahlungen zwischen beiden Systemen von dem kumulierten Marktwachstum, das über die Laufzeit jeweils linear modelliert wird. Marktdynamik (z.B. ein starkes Marktwachstum) steigert also den Wert eines flexibleren Systems. Das ist qualitativ unmittelbar einsichtig, kann mit Hilfe der vorgeschlagenen Methodik für ein gegebenes System jedoch auch quantifiziert werden. Die Ausreißer in sind dem Zusammenspiel aus Diskretisierung und jährlichem Bedarfswachstum geschuldet: Ein exaktes Aufeinandertreffen von Bedarf und Angebot sorgt insbesondere bei geringen Investitionsfixkosten für geringe kumulierte Auszahlungen.

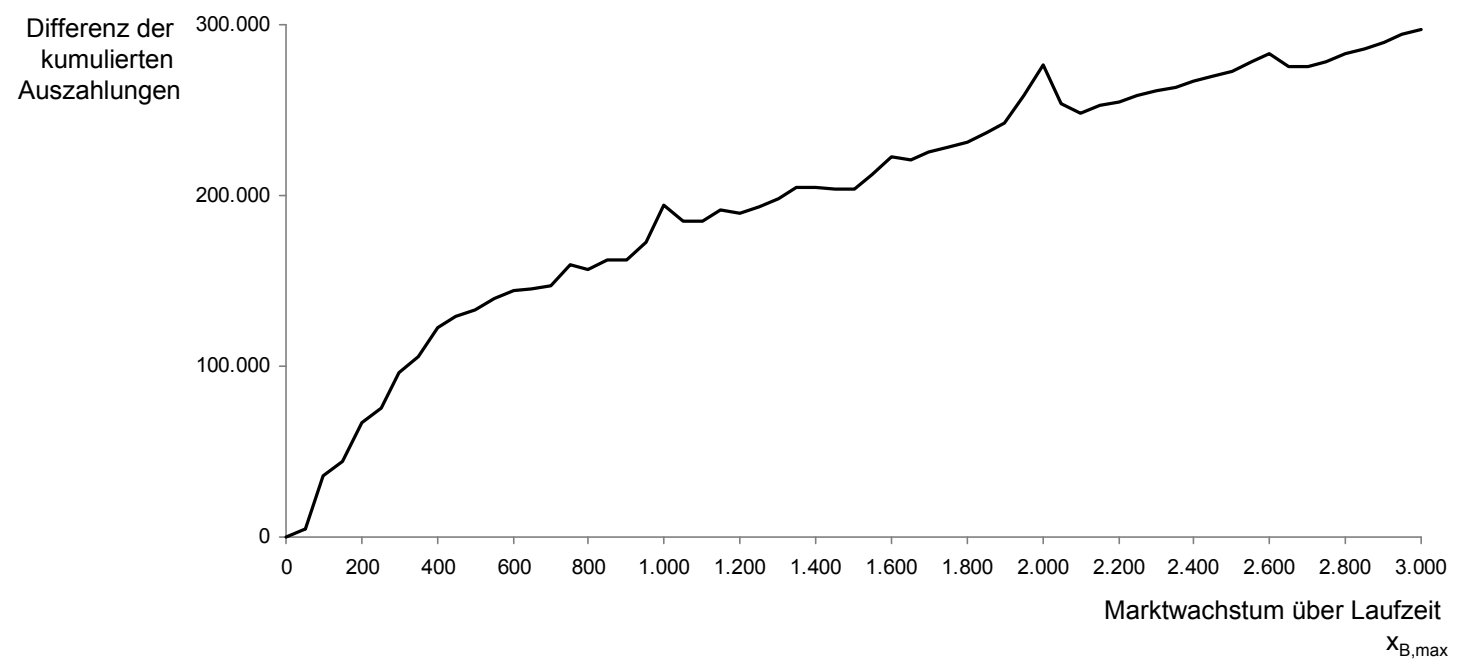

Abbildung 5: Sensitivitätsanalyse für kumulierte Auszahlungen in Abhängigkeit von Marktdynamik 


\section{Zusammenfassung und Ausblick}

Basierend auf den Anforderungen an die Quantifizierung von Effizienzgewinnen durch erhöhte Flexibilität intralogistischer Systeme wurde im vorliegenden Artikel eine Bewertungsmethodik vorgestellt, die sich für die Bewertung selbstorganisierter Systeme und des Internets der Dinge in der Intralogistik anbietet. Die Anwendung der Methode auf ein illustratives Beispiel zeigt die Notwendigkeit der vollständigen Analyse der Auszahlungen im Lebenszyklus; der offensichtliche Vorteil des flexibleren Systems in Form geringerer Investitionsfixkosten macht im Beispiel nur knapp 40\% der gesamten Effizienzgewinne aus. Weitere Vorteile ergeben sich aus geringeren Betriebskosten, geringeren Mehrkosten für Ersatztransporte und die im Durchschnitt späteren Investitionszeitpunkte. Erwartungsgemäß steigt der berechnete Wert der Flexibilität mit steigender Dynamik der Bedarfsentwicklung.

In weiteren Arbeiten werden wir die Theorie der vorgestellten Methodik weiter entwickeln und relevante praktische Beispiele untersuchen. Dabei soll auch der Zusammenhang zwischen Dynamik und Vorhersagbarkeit der Bedarfsentwicklung mit dem Wert flexibler Systeme näher beleuchtet werden. Letzteres scheint insbesondere auch für mittelständische Betriebe relevant, da diese weniger Möglichkeit haben, aufwändige Planungen und Bedarfsanalysen vorzunehmen.

\section{Literatur}

[Bengtsson01]

[Bengtsson02]

[Bretzke06]

[Böse07]

[Bullinger07]

[Chen96]

[Chryssolouris96]

[Copeland98]

[Corbey91]

[deMeyer98]

[deToni98]

[Domschke05]

[Freidenfels81]

[Günthner02]

[Günthner08a]
Bengtsson, Jens (2001): Manufacturing Flexibility and Real Options: A review. In: International Journal of Production Economics, H. 74, S. 213-224.

Bengtsson, Jens; Olhager, Jan (2002): Valuation of product-mix flexibility using real options. In: International Journal of Production Economics, H. 78, S. 13-28.

Bretzke, Wolf-Rüdiger (2006): Wettbewerb zwischen Supply Chains: Mehr Erfolg durch weniger Markt. In: Jacquemin, Mark; Pibernik, Richard; Sucky, Eric (Hg.): Quantitative Methoden der Logistik und des Supply Chain Management. Festschrift für Prof. Dr. Heinz

Böse, Felix; Windt, Katja (2007): Catalogue of Criteria for Autonomous Control in Logistics. In: Hülsmann, Michael; Windt, Katja (Hg.): Understanding Autonomous Cooperation and Control in Logistics. The Impact of Autonomy on Management, Information, Communication and Material Flow. Berlin Heidelberg New York: Springer, S. 57-72.

Bullinger, Hans-Jörg; ten Hompel, Michael (Hg.) (2007): Internet der Dinge. www.internet-der-dinge.de. Berlin Heidelberg New York: Springer

Chen, I. J.; Chung, C. -H (1996): An examination of flexibility measurements and performance of flexible manufacturing systems. In: International Journal of Production Research, Jg. 34, H. 2, S. 379-394.

Chryssolouris, G. (1996): Flexibility and Its Measurement. In: Annals of the CIRP, Jg. 45, H. 2, S. 581-587.

Copeland, Thomas E.; Keenan, Philip T. (1998): How much is flexibility worth. In: The McKinsey Quarterly, H. 2, S. 38-49.

Corbey, Michael (1991): Measurable economic consequences of investments in flexible capacity. In: International Journal of Production Economics, Jg. 23, S. 4757.

de Meyer, Arnoud (1998): Manufacturing Operations in Europe. Where Do We Go Next. In: European Management Journal, Jg. 16, H. 3, S. 262-271.

de Toni, A.; Tonchia, S. (1998): Manufacturing Flexibility: A Literature Review. In: International Journal of Production Research, Jg. 36, H. 6, S. 1587-1617.

Domschke, Wolfgang; Drexl, Andreas (2005): Einführung in Operations Research. 6. Auflage. Berlin Heidelberg New York: Springer.

Freidenfels, John (1981): Capacity Expansion. Analysis of Simple Models with Applications. New York: Elsevier.

Günthner, Willibald; Heinecker, Markus; Wilke, Michael (2002):

Materialflusssysteme für wandelbare Fabrikstrukturen. In: Industrie Management, Jg. 18, H. 5, S. 8-10.

Günthner, Willibald; Chisu, Razvan; Kuzmany, Florian (2008): Das "Internet der Dinge" - Teil I. Intelligent verteilt. In: F+H, H. 7-8, S. 422-425.

Günthner, Willibald; Chisu, Razvan; Kuzmany, Florian (2008b): Das "Internet der Dinge" - Teil II. Steuern ohne Hierarchie. In: F+H, H. 9, S. 494-497. 
[Günthner08b]

[Gupta93]

[Heinecker06]

[Hellingrath08]

[Hirschmeier05]

[Jedermann06]

[Kohagen08]

[Koste99]

[Kumar87]

[Möller08]

[Neumann77]

[Okujava06]

[Schlittgen01]

[Schuh06]

[Sethi90]

[Shuiabi05]

[Strüven04]

[tenHompel06]

[tenHompel08a]

[tenHompel08b]

[Upton94]

[Wilke06]
Günthner, Willibald; Chisu, Razvan; Kuzmany, Florian (2008): Internet der Dinge Teil III. Zukunftstechnologie mit Kostenvorteil. In: F+H, H. 10, S. 556-558.

Gupta, D. (1993): On measurement and valuation of manufacturing flexibility. In: International Journal of Production Research, Jg. 31, H. 12, S. 2947-2958.

Heinecker, Markus (2006): Methodik zur Gestaltung und Bewertung wandelbarer Materialflusssysteme. Dissertation. Technische Universität München, Lehrstuhl für Fördertechnik Materialfluss Logistik.

Hellingrath, Bernd; Schürrer, Sascha; Alberti, André; Krüger, Wiebke (2008): Der wahre Wert von RFID. In: Logistik Heute, Jg. 30, H. 5, S. 65-67.

Hirschmeier, Markus (2005): Wirtschaftlichkeitsanalysen für IT-Investitionen. Berlin: WiKu-Verlag.

Jedermann, Reiner; Gehrke, Jan D.; Lorenz, Martin; Herzog, Otthein; Lang, Walter (2006): Realisierung lokaler Selbststeuerung in Echtzeit. Übergang zum intelligenten Container. In: Pfohl, H. C.; Wimmer, T. (Hg.): Wissenschaft und Praxis im Dialog. Steuerung von Logistiksystemen - auf dem Weg zur Selbststeuerung. Hamburg: Deutscher Verkehrs-Verlag, S. 145-166.

Kohagen, Jens (2008): Dezentrale Intelligenz. In: Logistik inside, H. 6, S. 44-45. Koste, Lori L.; Malhotra, Manoj K. (1999): A Theoretical Framework for Analyzing the Dimensions of Manufacturing Flexibility. In: Journal of Operations Management, Jg. 18, S. 75-93.

Kumar, Vinod (1987): Entropic measures of manufacturing flexibility. In: International Journal of Production Research, Jg. 25, H. 7, S. 957-966.

Möller, Niklas (2008): Bestimmung der Wirtschaftlichkeit wandlungsfähiger Produktionssysteme. Dissertation. Technische Universität München, Lehrstuhl für Werkzeugmaschinen und Fertigungstechnik.

Neumann, Klaus (1977): Operations Research Verfahren. Dynamische Optimierung, Lagerhaltung, Simulation, Warteschlangen. 2 Bände. München Wien: Carl Hanser Verlag.

Okujava, Shota (2006): Wirtschaftlichkeitsanalysen für IT-Investitionen. Ein kontinuierlicher und stakeholderorientierter Ansatz. Duisburg: WiKu-Verlag. Schlittgen, Rainer; Streitberg, Bernd H. J. (2001): Zeitreihenanalyse. München: Oldenbourg.

Scholz-Reiter, Bernd; Schulz, Stephan (2007): Selbststeuerung in der Logistik. In: Wolf-Kluthausen, Hanne (Hg.): Jahrbuch Logistik 2007. Korschenbroich: free Beratung GmbH , S. 236-240.

Schuh, Günther; Wemhöner, N.; Friedrich, Ch (2006): Scenario-based Lifecycle Analysis of Manufacturing Systems. In: CIRP - Journal of Manufacturing Systems, Jg. 35, H. 2.

Sethi, Andrea Krasa; Sethi, Suresh Pal (1990): Flexibility in Manufacturing: A Survey. In: The International Journal of Flexible Manufacturing Systems, H. 2, S. 289-328.

Shuiabi, Eyas; Thomson, Vince; Bhuiyan, Nadia (2005): Entropy as a Measure of Operational Flexibility. In: European Journal of Operational Research, Jg. 165, S. 696-707.

Slack, Nigel (1983): Flexibility as a Manufacturing Objective. In: International Journal of Operations \& Production Management, Jg. 3, H. 3, S. 4-13.

Strüven, Peter; Spettmann, Ralf (2004): Produktionsstandort Deutschland - quo vadis. Studie, The Boston Consulting Group.

ten Hompel, Michael (2006): Zellulare Fördertechnik. In: Logistics Journal. ten Hompel, Michael; Nagel, Lars (2008): Zellulare Transportsysteme. Den Dingen Beine machen im "Internet der Dinge". In: it - Information Technology, Jg. 50, H. 1, S. 59-65.

ten Hompel, Michael; Follert, Guido; Roidl, Moritz (2008): Künstliche Intelligenz im Internet der Dinge: Die Zukunft der Materialflusssteuerung mit autonomen Agenten. In: Wolf-Kluthausen, Hanne (Hg.): Jahrbuch Logistik 2008.

Korschenbroich: free Beratung GmbH , S. 24-29.

Upton, David M. (1994): The Management of Manufacturing Flexibility. In: California Management Review, Jg. 36, H. 2, S. 72-89.

Wilke, Michael (2006): Wandelbare automatisierte Materialflusssysteme für dynamische Produktionsstrukturen. Dissertation. Technische Universität München, Lehrstuhl für Fördertechnik Materialfluss Logistik. 
[Windt08]

Windt, Katja (2008): Ermittlung des angemessenen Selbststeuerungsgrades in der Logistik. In: Nyhuis, Peter (Hg.): Beiträge zu einer Theorie der Logistik. Berlin Heidelberg: Springer, 350-372. 